\title{
Agent-based Modeling Simulation Analysis on the Regulation of Institutional Investor's Encroachment Behavior in Stock Market
}

\author{
Yang $\mathrm{Li}^{1}$, Shuo $\mathrm{Liu}^{2,3}$ \\ ${ }^{1}$ School of Economics and Management, Beijing Institute of Petrochemical Technology (China) \\ ${ }^{2}$ School of Economics \& Management, Beijing Institute of Graphic Communication (China) \\ ${ }^{3}$ China Center for Industrial Security Research, Beijing Jiaotong University (China) \\ livangbd@,163.com, liusbuo621@,163.com
}

Abstract:

Purpose: This study explores the effective regulation of institutional investor's encroachment behavior in stock market. Given the theoretical and practical importance, the present study examines the effect of the self-adaptive regulation strategy (adjusting the regulation factors such as punishment and the probability of investigating successfully in time) for the sake of the small \& medium-sized investor protection.

Design/methodology/approach: This study was carried out through game theory and agent-based modeling simulation. Firstly, a dynamic game model was built to search the core factors of regulation and the equilibrium paths. Secondly, an agent-based modeling simulation model was built in Swarm to extend the game model. Finally, a simulation experiment (using virtual parameter values) was performed to examine the effect of regulation strategy obtained form game model.

Findings: The results of this study showed that the core factors of avoiding the institutional investor's encroachment behavior are the punishment and the probability of investigating successfully of the regulator. The core factors embody as the self-adaptability and the capability 
of regulator. If the regulator can adjust the regulation factors in time, the illegal behaviors will be avoided effectively.

Research limitations/implications: The simulation experiment in this paper was performed with virtual parameter values. Although the results of experiment showed the effect of selfadaptive regulation, there are still some differences between simulation experiment and real market situation.

Originality/value: The purpose of this study is to investigate an effective regulation strategy of institutional investor's encroachment behavior in stock market in order to maintain market order and protect the benefits of investors. Base on the game model and simulation model, a simulation experiment was preformed and the result showed that the self-adaptive regulation would be effective. This study applied game theory and agent-based modeling simulation to the research of financial regulation, and extended the application field of these two methods.

Keywords: institutional investor, agent-based modeling, simulation, regulation, self-adaptability

\section{Introduction}

Stock market is the most important and active component part of investment market in one country. There are several kinks of investors trading in stock market in order to make a profit, institutional investors and small \& medium-sized investors are the two important kinds of market participants. Although institutional investors are few in number, they are powerful enough to affect the price movements of one or several stocks through stock trading or affecting the listed companies' operation; small \& medium-sized investors are numerous, but they are always the price takers of stock. Based on the properties of institutional investors, they can encroach on the small \& medium-sized investors' benefits easily. For the sake of investors' benefits especially the small \& medium-sized investors', the regulator of stock market is searching the scientific regulation strategies.

This paper studies the regulation strategies for one kind of illegal behavior which is the encroachment on small \& medium-sized investors' benefits carried out by institutional investor through game theory and agent-based modeling simulation. Firstly, a dynamic game model is built to analyze the equilibrium paths and the relationship among the institutional investor, small \& medium-sized investors and the regulator. Secondly, an agent-based modeling simulation model is built in order to test the results of game analysis. Finally, we propose a self-adaptive regulation strategy to avoid the encroachment behavior on small \& medium-sized investors. 


\section{Literature review}

Today, financial security has become a key problem for every country. Other studies have been mainly focus on the laws and ignored the behavioral features of the participants in financial market. As the computer science has been highly developed, the researcher may build virtual financial system to research the participants and the system evolution in computer environment. Since then, the simulation is becoming an effective method for financial security and policy research. Bruun and Luna (1999) built an agent-based modeling simulation model for simulating the macro-economical operation in Swarm environment and research the behavioral features of macro-economical participants. And their model proved that the simulation model could simulate the reality quite well. Luna and Stefannson (2001) compiled an academic monograph based on a series of simulation researches and discussed the feasibility of simulation method for financial research. And they explained how to program the simulation model in Swarm environment, which makes their monograph be an important reference for agent-based modeling simulation research. Foster (2005) investigated the complex system in depth and proposed that the complex system research should consider the relationship among the participants. Foster's research required the genetic algorithm to endow simulation agents with self-adaptability in order to make the simulation model be close to the reality as far as possible. Ren (2006) investigated the regulation of insurance market through game theory and agent-based modeling simulation in Swarm. The simulation experiments showed that the regulator can control the illegal behaviors through controlling the key parameters of insurance applicants. Liu and Han (2007) established a multi-agent artificial stock market in order to study the characteristic of investment behavior of investors. The simulation experiments showed that the investors would choose the stock for maximizing investment revenue based on their wealth and investment experiences. Liu, Zhang and $\mathrm{He}$ (2008) proposed a multi-agent simulation framework based on finite automaton for studying the bounded rationality behaviors of economic agents. This simulation framework could carry out the simulation of sequential game and evolutionary game. A series simulation experiments for repeated prisoners' dilemma with three players were carried out, which verified the availability of the multi-agent simulation framework. Tedeschi, Iori and Gallegati (2009) studied the market effect of the exchange of information among investors and investment behaviors imitation in their simulation model. Their research brought new ideas for simulating the behaviors of investors and the information flows. Liu (2010) analyzed the possibility of the institutional investors' conspiring to control some new stock in Chinese stock market based on game theory and simulation model. An agent-based simulation model in Swarm was built to research the investment behaviors of institutional investors and the regulation of the illegal phenomenon. Some scientific measures to avoid the conspiring control on the new stock were proposed based on the game and simulation analysis. Liu and Wang (2011) studied the regulation of institutional investors encroaching on small and medium- sized investors' benefit in stock market through establishing a static game model and its simulation model in Swarm. 
Severe beforehand- regulation, faultless company management and smarter regulator would avoid the encroaching of institutional investors based on the game analysis and simulation experiments.

The following game and simulation analysis are based upon the previous research results.

\section{Game analysis}

\subsection{Description of the game}

Because the institutional investor has plenty of capital, so he can own most of circulation stocks of one listed company, which creates the condition for him to control the company and its wealth. In this paper we assume that the institutional investor will encroach on the small \& medium-sized investors' benefits through encroaching on the wealth of the listed company. To avoid this kind of illegal behavior, the regulator of stock market should take necessary measures. From the description of the game, we know that there are three kinds of players involved in the game, which are institutional investor, small \& medium-sized investors and regulator.

The game has three steps. Firstly, the institutional investor chooses to encroach or not to. If the institutional investor decides to encroach on the wealth of listed company, he will own "a" percents of the total circulation stocks and encroach on ratio "B" of listed company's wealth "W"; and the institutional investor will take cost " $\mathrm{C}_{\mathrm{e}}$ " to do this. If institutional investor chooses not to encroach, nothing happens. The second step of the game is in small \& medium-sized investors' turn. The small \& medium-sized investors will choose to complain or not to complain when their benefits are encroached on. If the small \& medium-sized investors choose not to complain, they will lose "(1-a) $\bullet B \bullet W "$ which will be the illegal profit of the institutional investor; if they do complain they will pay " $C_{p}$ " as the cost and the game will enter the third step. In the third step of the game, the regulator will choose to investigate or not to. If the regulator chooses to investigate the encroachment behavior, he will pay " $C_{R}$ " as the cost. If the regulator investigates the illegal behavior successfully, the institutional investor will face punishment "N", compensation "PF" and encroaching income "(1-a) •B•W" given back to small \& medium-sized investors; and the regulator will gain " $U$ " including good reputation and so on. If the regulator investigates unsuccessfully or chooses not to investigate, the institutional investor will get away with murder and the small \& medium-sized investors will bear the loss, and the regulator will get bad reputation "- $U$ " as its negligence of duty simultaneously. In conclusion, this is a dynamic game with complete information and the game tree is shown as Figure 1. 


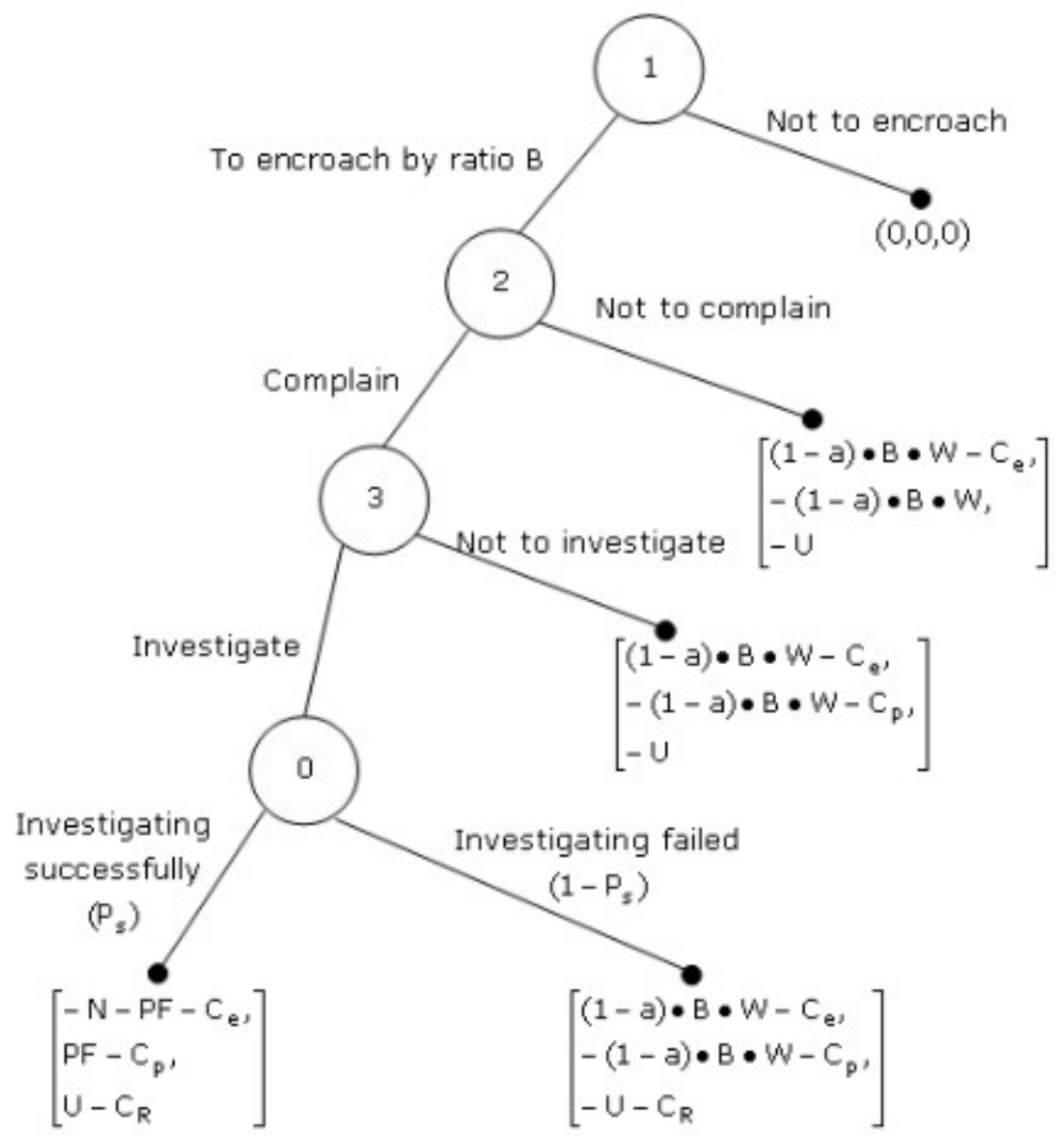

Figure 1. The structure of the game

In the game tree described in Figure 1, player 1 stands for institutional investor; player 2 stands for the small \& medium-sized investors; player 3 stands for the market regulator; player 0 is the virtual player "Nature" who will decide the probability of investigating successfully or failed.

\subsection{Equilibrium paths of the game}

From the description and game tree, we have known that it is a dynamic game with complete information, and backward induction method is the effective measure for solving the subgame perfect Nash equilibrium.

Firstly, we start from the third step of the game, which is the regulator's decision. The better result for the market and small \& medium-sized investors is that the regulator chooses to investigate the encroachment behavior. The necessary condition of investigating is the profits regulator gained when he investigates bigger than he does not. IF the virtual player "Nature" 
sets the probability of investigating successfully or failed as "( $\left.P_{s}, 1-P_{s}\right)$ ", the condition will be shown as formula (1).

$$
P_{s} \bullet \cdot\left(U-C_{R}\right)+\left(1-P_{s}\right) \bullet\left(-U-C_{R}\right)>-U
$$

Secondly, we go back to the second step of the game, which is the small \& medium-sized investors' decision. The better result for the stock market is that the small \& medium-sized investors choose to complain. The necessary condition of this result is the profits small \& medium-sized investors gained when they complain bigger than they do not; the condition will be shown as formula (2).

$$
P_{s} \bullet\left(P F-C_{p}\right)+\left(1-P_{s}\right) \bullet\left[-(1-a) \bullet B \bullet W-C_{p}\right]>-(1-a) \bullet B \bullet W
$$

Finally, we go back to the first step of the game, which is the institutional investor's decision. When the condition shown as formula (3) is satisfied, the institutional investor will choose to encroach on.

$$
P_{s} \bullet\left(-N-P F-C_{e}\right)+\left(1-P_{s}\right) \bullet\left[(1-a) \bullet B \bullet W-C_{e}\right]>0
$$

On the contrary, the institutional investor will choose not to encroach on the small \& mediumsized investors' benefits; the condition is shown as formula (4).

$$
P_{s} \bullet\left(-N-P F-C_{e}\right)+\left(1-P_{s}\right) \bullet\left[(1-a) \bullet B \bullet W-C_{e}\right]<0
$$

To sum up the solution, we acquire two equilibrium paths, one is "(To encroach, Complain, Investigate)" and the other is "(Not to encroach)". From formula (1) to formula (4), we know that there are three key factors which are " $\mathrm{P}_{\mathrm{s}}$ ", "N" and "PF". Factor " $\mathrm{P}_{\mathrm{s}}$ " reflects the capability of regulator, "N" stands for the punishment given to the institutional investor from regulator, and "PF" which stands for the compensation for small \& medium-sized investors. And we can deduce that the three regulation factors are the bigger the better. When the three regulation factors are big enough, the best equilibrium path that "(Not to encroach)" will be achieved. 
Based on the game analysis, we will build a dynamic agent-based modeling simulation model in Swarm environment in order to test the results of game analysis and propose the selfadaptive regulation strategy.

\section{Agent-based modeling simulation model in Swarm}

\subsection{Agents of the simulation model}

There are two kinds of agents in the simulation model of Swarm, one kind is simulation agent and the other kind is functional agent. The simulation agent is the mapping of game player. The functional agent is invisible in game model but it will endow the simulation agent with extra attributes. Based on the game model discussed above, there are three simulation agents who are institutional investor (Iinvestor), small \& medium-sized investors (MSinvestor) and regulator (Regulator) in the simulation model. In addition, the simulation model has one functional agent which is Genetic Algorithm Learning Classifier System (GALCS). The function of GALCS is to endow the simulation agents with self-adaptability.

As the simulation model is the extension of game model and the simulation agents are the mappings of game players, the simulation agents should have the properties and actions as they have in game model. The main properties and actions of simulation agents are described in Table1 and Table 2.

\begin{tabular}{|l|l|l|}
\hline \multirow{2}{*}{ Agent } & Property & $\begin{array}{l}\text { Variable in simulation } \\
\text { model }\end{array}$ \\
\hline \multirow{4}{*}{ Iinvestor } & The encroachment ratio & B \\
\cline { 2 - 3 } & The profit of current simulation cycle & profit \\
\cline { 2 - 3 } & The profit of last simulation cycle & lastprofit \\
\hline \multirow{4}{*}{ Regulator } & The probability of investigating successfully & PS \\
\cline { 2 - 3 } & The punishment index & $\mathrm{N}$ \\
\cline { 2 - 3 } & The profit of current simulation cycle & profit \\
\cline { 2 - 3 } & The profit of last simulation cycle & lastprofit \\
\hline \multirow{3}{*}{ MSinvestor } & The profit of current simulation cycle & profit \\
\cline { 2 - 3 } & The profit of last simulation cycle & lastprofit \\
\cline { 2 - 3 } & Public information & Wealth \\
\hline
\end{tabular}

Table 1 . The main properties of the simulation agents 


\begin{tabular}{|c|l|l|l|}
\hline \multirow{4}{*}{ Agent } & \multicolumn{1}{|c|}{ Action } & Function description & Action type \\
\hline \multirow{5}{*}{ Regulator } & -encroach & $\begin{array}{l}\text { Deciding the change of the encroachment ratio B } \\
\text { according to the regulation situation }\end{array}$ & Simulation action \\
\cline { 2 - 4 } & -compare & $\begin{array}{l}\text { Computing the profit in current simulation cycle } \\
\text { and providing evidence for modifying strategy in } \\
\text { the next cycle }\end{array}$ & Simulation action \\
\cline { 2 - 5 } & -setPS & $\begin{array}{l}\text { Deciding the change of the probability of } \\
\text { investigating successfully PS according to the } \\
\text { illegal situation }\end{array}$ & Simulation action \\
\cline { 2 - 5 } & -compare & $\begin{array}{l}\text { Deciding the change of the punishment index N } \\
\text { according to the illegal situation }\end{array}$ & Simulation action \\
& and providing evidence for modifying strategy in & Simulation action \\
\hline \multirow{2}{*}{ MSinvestor } & -compute & Computing the expect profit & Simulation action \\
\cline { 2 - 5 } & -get & Getting the public informations & Functional action \\
\hline
\end{tabular}

Table 2. The main actions of the simulation agents

\subsection{Genetic Algorithm Learning Classifier System}

Genetic Algorithm Learning Classifier System (GALCS) is a very important agent in the simulation model. Different from one time game, the dynamic simulation will run the game process repeatedly as the market realities. In the real stock market, the participants will optimize their decisions in real time. In order to make the simulation model conform to the market realities as far as possible, the simulation agents need self-adaptability to adjust their decisions. GALCS is the functional agent which will endow the simulation agents with selfadaptability. The GALCS will accumulate the probability of optimal decision when the simulation agents face the same situation as before. In this simulation model, institutional investor agent (Iinvestor) and regulator agent (Regulator) are endowed with self-adaptability by GALCS.

The GALCS of institutional investor agent (Iinvestor) uses the probability of successful investigation " $\mathrm{P}_{\mathrm{s}}$ " and punishment from regulator " $\mathrm{N}$ " as the adjustment coefficients, which both have three statuses including rising, flatting and declining. So the institutional investor agent will face $3 \times 3=9$ different situations. The institutional investor will adjust his GALCS according to his profit changing, when his profit rises he will increase the cumulative probability of the current decision, on the contrary, he will decrease the cumulative probability of the current decision if he faces the same situation in the future. The GALCS of regulator agent (Regulator) uses the encroachment ratio "B" and the profit of institutional investor agent as the adjustment coefficients, which also both have three statuses, and the regulator agent will face 9 different situations too. Same as the GALCS of institutional investor, the regulator agent will optimize his decision according to his own profit changing. By doing so, the institutional investor agent and regulator agent will optimize their decisions according to the situation, and we can research how the equilibrium paths generate and the effect of selfadaptive regulation strategy. 


\subsection{Environment of the simulation model}

According to the relationship among the simulation agents and functional agent, the environment of the simulation model may be described as Figure 2. In Figure 2, Iinvestor and Regulator are the mapping of game players; the GALCS is the functional agent who endows the simulation agents with self-adaptability. ModelSwarm is a functional agent to control the simulation process in Swarm.

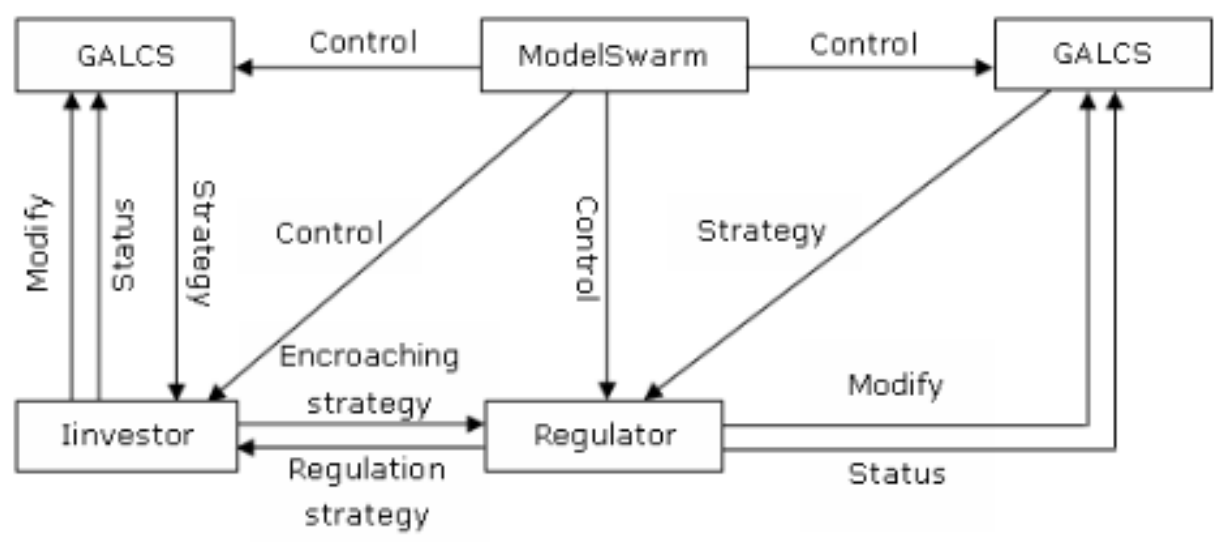

Figure 2. The environment of the simulation model

\subsection{The action sequence of the simulation agents}

The last work of building a simulation model is to define the action sequence of the simulation agents in one simulation cycle. There are seven steps in this simulation model. The simulation process and information flows are shown as Figure 3.

The first step: The institutional investor agent (Iinvestor) performs his action named "-encroach" to decide the change of the encroachment ratio "B" according to the regulation situation.

The second step: The small \& medium-sized investors agent (MSinvestor) performs his action named "-compute" to compute the expect profit he will gain or lose.

The third step: The regulator agent (Regulator) performs his action named "-setPS" to decide the change of the probability of investigating successfully "Ps" according to the illegal situation.

The fourth step: The regulator agent (Regulator) performs his action named "-setN" to decide the change of the punishment index " $\mathrm{N}$ " according to the illegal situation. 


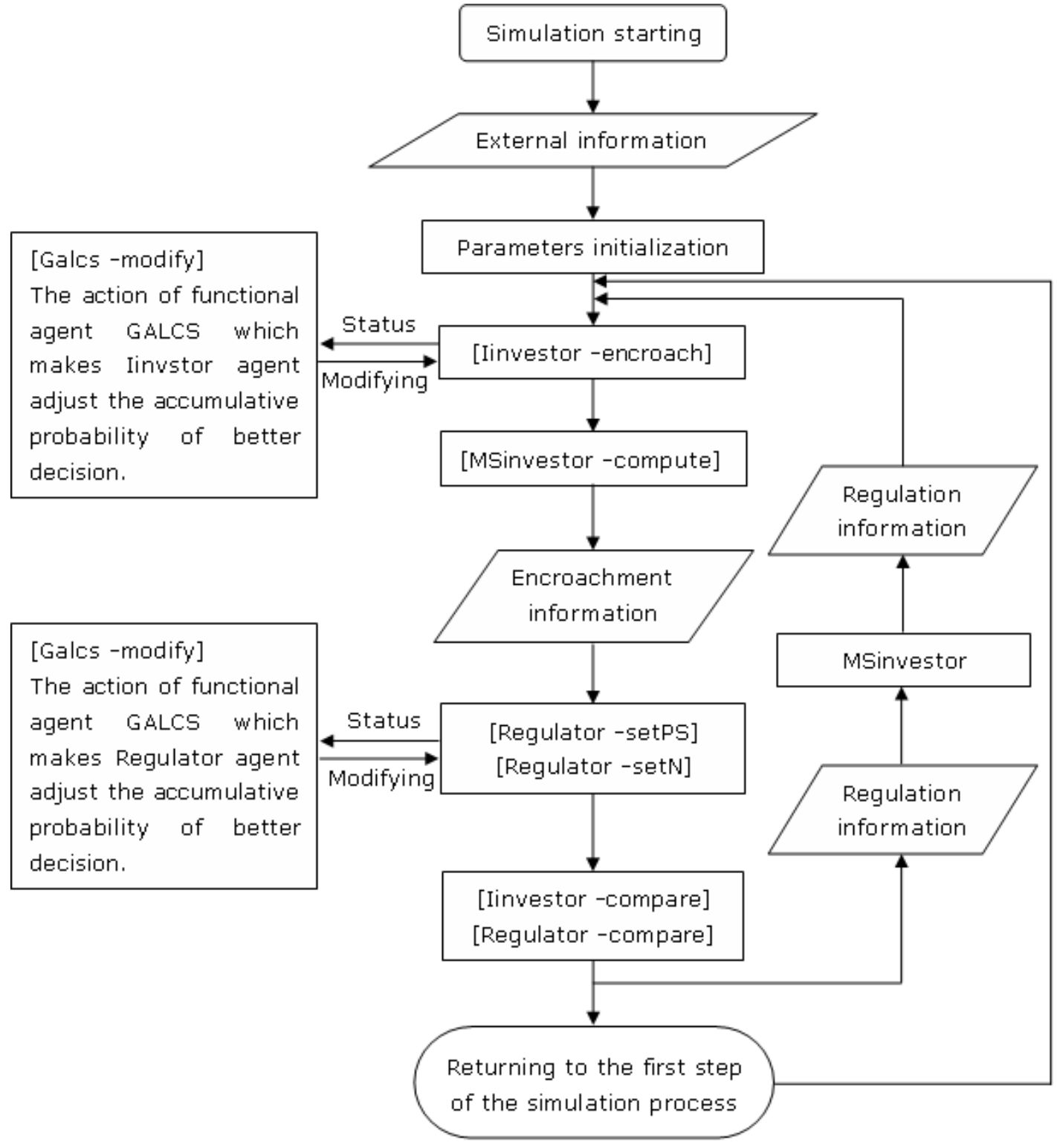

Figure 3. The process and information flows of the simulation model

The fifth step: The institutional investor agent (Iinvestor) performs his action named "compare" to compute the profit in current simulation cycle and provide evidence for modifying encroachment strategy in next cycle.

The sixth step: The regulator agent (Regulator) performs his action named "-compare" to compute the profit in current simulation cycle and provide evidence for modifying regulation strategy in next cycle.

The seventh step: One whole simulation cycle is over; the simulation process will go back to the first step. 


\subsection{Simulation experiment}

After running the simulation model, a dialog box shown as Figure 4 for parameters initialization will be shown to us.
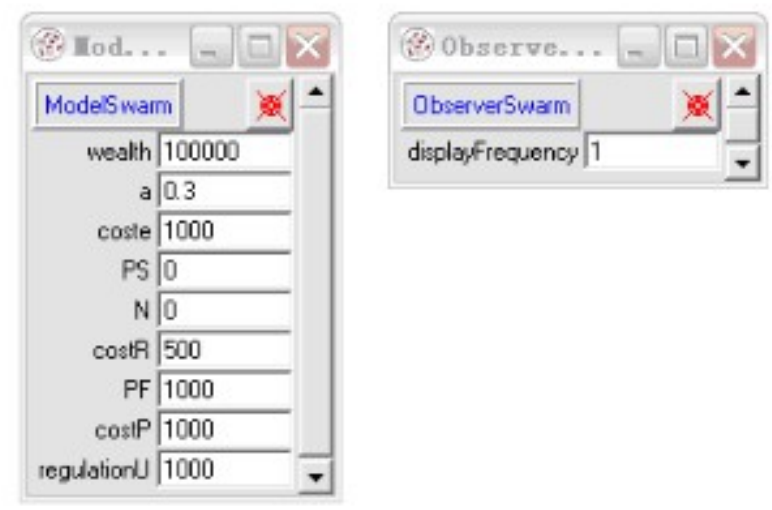

Figure 4. The dialog box for parameters initialization

There are nine parameters as the mapping of game model need to be initialized including the wealth of the listed company "wealth"; the ratio of the circulation stock institutional investor held "a"; the cost for the encroachment behavior of institutional investor "coste"; the probability of investigating successfully of regulator "PS"; the punishment index for the institutional investor " $\mathrm{N}$ "; the compensation for the small \& medium-sized investors "PF"; the cost of complaining for the small \& medium-sized investors "costP"; the utility as good reputation gained by regulator when investigating successfully "regulationU". These nine parameters will be initialized as $100000,0.3,1000,0,0,500,1000,1000$ and 1000. The initial values of parameters are all the virtual values, and using virtual values will not affect the research. The simulation experiment will proceed in two phases, one phase will run the simulation model with no regulation, and the other phase will run the simulation model with self-adaptive regulation strategy. We will research the decision properties of institutional investor in order to investigate the effect of self-adaptive regulation strategy.

The first experiment phase is the situation that the regulator has no regulation strategy against the encroachment behavior, and the result is shown in Figure 5. When the regulator has no regulation strategy, the encroachment ratio "B" of institutional investor will increase rapidly and the ratio "B" will keep in $100 \%$ after some time. The result means that the institutional investor will do his illegal behavior unscrupulously and the listed company and the small \& medium-sized investors will lose lots of wealth at the same time. In order to protect the benefits of small \& medium-sized investors and maintain the market order, the effective regulation strategy is necessary. 


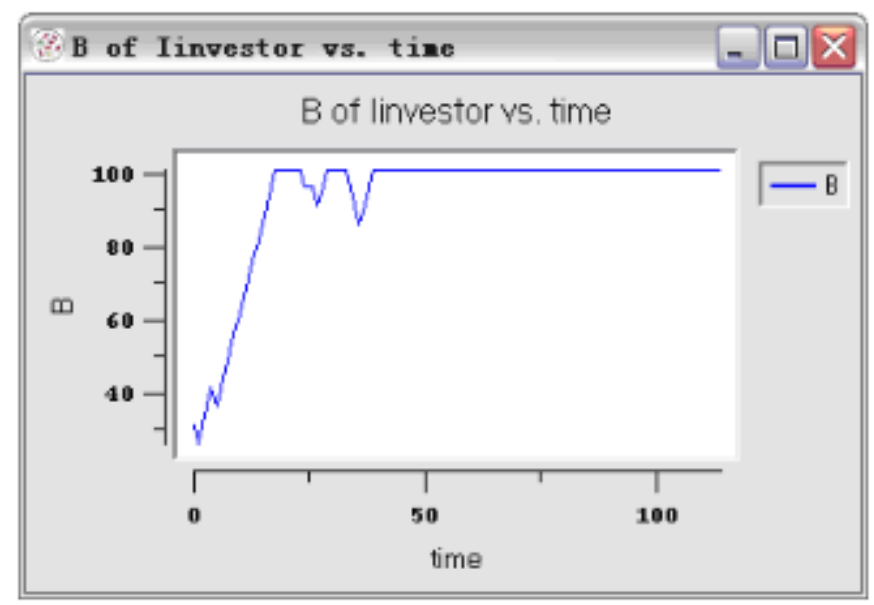

Figure 5 . The change of encroachment ratio $B$ without regulation

The second experiment phase is the situation that the regulator uses the self-adaptive regulation strategy. The main character of the self-adaptive regulation is the regulator can adjust his regulation factors which are "PS" and "N" timely according to the situation. The simulation result is shown in Figure 6.

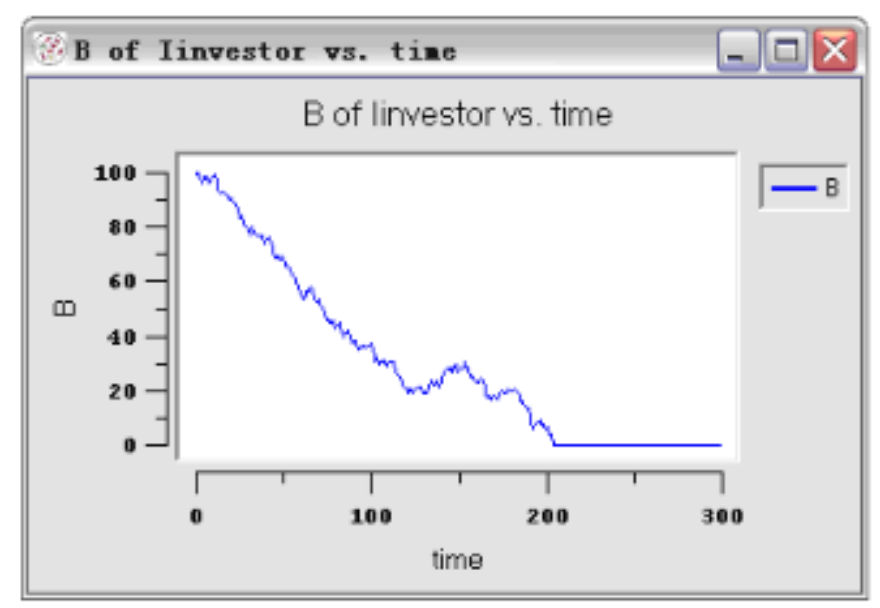

Figure 6 . The change of encroachment ratio $B$ with self-adaptive regulation

As the regulator has self-adaptability, he will adjust his regulation factors timely according to the institutional investor's decision; and the institutional investor will adjust his encroachment strategy according to the regulation situation. At the beginning, we see that when the regulation is not strictly, the institutional investor will encroach on the wealth by a high ratio "B". In order to avoid the bad reputation and profit loss, the regulator will strengthen the regulation by increase his capability "PS" and the punishment index "N" timely. By doing so, the institutional investor will face great loss and the only way to avoid this is to decrease the encroachment ratio until reduced to zero. When the encroachment ratio "B" drops to zero, the 
best game equilibrium path that "(Not to encroach)" will be achieved, and the institutional investor will not do the illegal behavior.

\section{Conclusion}

From the discussion above, it's not hard to see that the game analysis is a kind of one time analysis of strategies, but the simulation model based on the game model is a dynamic and repeated process of the game. So the simulation model will show the adjustment of players or agents' strategies and how the equilibrium paths generate. In game analysis, we know that the probability of investigating successfully " $\mathrm{P}_{\mathrm{s}}$ " which reflects the capability of regulator, the punishment "N", and the compensation for small \& medium-sized investors "PF" are the key regulation factors of avoiding the encroaching performed by the institutional investor. The bigger the three factors are the more likely to avoid the illegal behavior carried out by institutional investor. According to market realities and game model, we use a functional agent named GALCS to endow the players with self-adaptability which makes the investors and regulator agents optimize their strategies timely. In the simulation model, we know that the best regulation strategy for regulator is his self-adaptation. In order to increase the profit, the regulator must improve his capability which reflected as " $\mathrm{P}_{\mathrm{s}}$ " and adjust the punishment " $\mathrm{N}$ ". When the regulator has a high " $\mathrm{P}_{s}$ " and "N", the institutional investor will choose not to encroach on the benefits of small \& medium-sized investors, and the best equilibrium path will be achieved.

\section{References}

Bruun, C., \& Luna, F. (1999). Endogenous Growth in a Swarm Economy: Fighting Time, Space, and Complexity. Computing in Economics and Finance from Society for Computational Economics, 1354.

Foster, J. (2005). From Simplistics to Complex Systems in Economics. Cambridge Journal of Economics, 29, 5-14. http://dx.doi.org/10.1093/cje/bei083

Liu, S. (2010). Agent-based Simulation Analysis of Regulation of Institutional Investors Investing on The Initial Public Offerings. Special Zone Economy, 9, 121-122.

Liu, S., \& Wang, W.J. (2011). Agencies Violations Small Investors Illegal Regulatory Game Simulation. Techno-economics \& Management Research, 6, 3-6.

Liu, W.N., \& Han, L.Y. (2007). Simulation of Investors by Multi-Agent Artificial Stock Market Model. Chinese Journal of Management, 4, 414-420. 
Liu, Z., Zhang, X.L., \& HE, J.K. (2008). Study on Simulation Frame for Multi-agent Game in Experimental Economics. Journal of System Simulation, 20, 6658-6663.

Luna, F., \& Stefannson, B. (2001). Economic Simulation in Swarm: Agent-Based Modeling Object Oriented Programming. Kluwer Academic Publishers.

Ren, T. (2006). A simulating test of an insurance game analyzing model. Research on Economics and Management, 11, 84-89.

Tedeschi, G., Iori, G., \& Gallegati, M. (2009). The role of communication and imitation in limit order markets. The European Physical Journal B-Condensed Matter and Complex Systems, 71, 489-497. http://dx.doi.org/10.1140/epjb/e2009-00337-6

Article's contents are provided on a Attribution-Non Commercial 3.0 Creative commons license. Readers are allowed to copy, distribute and communicate article's contents, provided the author's and Journal of Industrial Engineering and Management's names are included. It must not be used for commercial purposes. To see the complete license contents, please visit http://creativecommons.org/licenses/by-nc/3.0/. 